\title{
DEFECT FORMATION DURING SYNTHESIS AND WELDING OF SILVER NANOWIRES FOR SOLAR CELL APPLICATIONS
}

\author{
Vuk V. Radmilović* and Velimir R. Radmilović \\ University of Belgrade, Faculty of Technology and Metallurgy, \\ Karnegijeva 411120 Belgrade, Serbia
}

Received 25.10.2019

Accepted 01.01.2020

\begin{abstract}
Structural defects such as stacking faults and twins, observed in many facecentered cubic (FCC) structured metals and alloys with low stacking fault energies, in general, play an important role in microstructure evolution, and in particular, in nucleation and crystal growth, morphology development, and phase transformations during synthesis as well as post-synthesis processes.
\end{abstract}

Keywords: defect formation; welding of silver nanowires, solar cell.

\section{Introduction}

Solar cells, especially next-generation devices, exhibit huge potential in helping to reach the goal of completely switching to renewable energy sources on a global level. The work presented in this paper was focused on silver nanowire (AgNW) networks for application as a transparent electrode layer in solar cells. These devices, also known as photovoltaics, usually are multilayered systems, where the active layer is the most important as it is the place where the actual photo conversion occurs, i.e. solar energy is converted into electrical energy through the photovoltaic effect. Electrodes in solar cells also serve an important role as they are selective contacts which form differences in electrical potential. This helps dissociate excitons (electron-hole pairs), i.e. charge carriers, and in turn guides them to their respective electrodes and hence creates an electrical current. Among all materials researched so far, indium tin oxide (ITO), a degenerately doped n-type semiconductor, has been proven to be most effective as a transparent electrode in the next generation of solar cells, mainly due to its optimal optoelectronic characteristics - low electrical sheet resistance and high optical transparency. Despite these beneficial properties, alternative materials need to be explored because of several drawbacks: high prices of indium, expensive and demanding (high-temperature, high-vacuum and inert conditions) processing techniques, lack of

*Corresponding author: Vuk V. Radmilović, vukradmilovic@tmf.bg.ac.rs 
mechanical flexibility (brittleness), etc. One of the most promising alternatives are nanowire networks, due to their excellent optoelectronic properties, second only to ITO [1]. Their advantage is that they can be processed from solution giving them the potential for being a low-cost alternative to sputtered or thermally evaporated metal films.

The electrical properties of AgNW networks result from the dimensions of individual AgNWs as well as the morphology of the network itself, e.g. electrical resistance increases as the length and diameter of the NWs decrease. This is due to the surface scattering of electrons [2]. This relation is very complex as the percolation of the network has a significant role, i.e. when two NWs are in contact with one another, they are touch through a junction, extending the percolation of the network on one hand, but increasing electrical resistance on the other hand, as junctions exhibit enormous resistances of up to $1 \mathrm{G} \Omega$, much higher than in the AgNWs themselves [3]. With this in mind, it is clear that longer AgNWs require fewer junctions for creating percolation [4] with the minimum number of NWs required to produce a conductive path across the network being proportional to the square of the NW lengths [5]. As junction resistance between the AgNWs is very high, certainly affecting the resistance of the entire network, various post-processing methods have been implemented in order to modify the junction morphology thereby reducing the resistance of the entire network without affecting optical properties, including thermal annealing [6], plasmonic welding, inducing local melting and solidification [7], high-intensity pulsed light technique (HIPL) [8], plasma sintering at elevated [9] and room temperatures [10], cold rolling [11] and chemical approach by ion exchange [12].

This paper aimed to investigate microstructure evolution and the formation of defects such as twins and stacking faults [13, 14], during the synthesis of AgNWs as well as welding by annealing, performed in order to improve conductivity without sacrificing transparency of the electrode layer [15]. The incorporation of $\mathrm{Ag}$ atoms is driven by surface diffusion and the tendency to decrease surface energy [16]. This means that control of the kinetics will influence the final morphology of welded AgNW junctions.

\section{Experimental}

Synthesis of AgNWs was done by the polyol reduction method, where ethylene glycol (EG) was used simultaneously as a reducing agent and solvent while silver nitrate $\left(\mathrm{AgNO}_{3}\right)$ was introduced as a silver precursor [17]. The polymer polyvinylpyrrolidone (PVP) was used as a surfactant whose role was to shape of AgNWs during synthesis by preventing coalescence of nuclei during initial growth [18]. This was achieved by binding of PVP to $\{100\}$ AgNW facets which lead to their passivation enabling the growth of only $\{111\}$ facets in the [110] direction $[19,20]$ resulting in a one-dimensional (1D) structure.

In order to reduce sheet resistance, the AgNW network was annealed at $250^{\circ} \mathrm{C}$ for 3 minutes and subsequently coated by a layer of aluminum-doped zinc oxide (AZO) for the enhancement of properties essential for an electrode in a solar cell. Besides being a wide bandgap semiconductor, AZO when coupled with AgNWs as a nanocomposite, exhibits greater charge carrier collection, improved chemical and mechanical stability. This, unfortunately, comes with drawbacks as joining AZO with AgNWs leads to optical transmittance decrease and reflection increase due to scattering of light from AgNWs into 
the AZO. The detailed experimental procedure can be found in our previous publication [15].

For an in-depth comprehension of morphology and microstructure of AgNWs and AgNW/AZO nanocomposite, scanning electron microscopy (SEM) as well as conventional (CTEM) and scanning transmission electron microscopy (STEM) in tandem with energy dispersive spectroscopy (EDS), were used. Sample preparation for transmission electron microscopy consisted of obtaining cross-sectional samples by lifting out lamellae by focused ion beam (FIB) machining inside the dual-beam SEM/FIB system, in the manner shown in Figure 1, and transferring the lamellae into the TEM for final characterization.

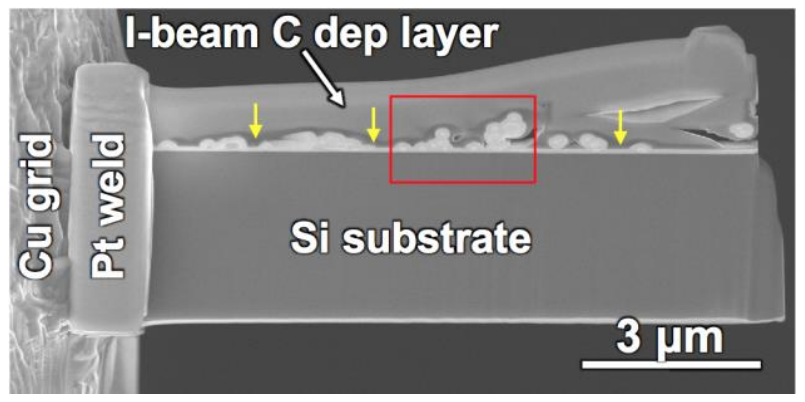

Fig. 1. Scanning electron micrograph of AgNW/AZO cross-section lamella sample; yellow arrows indicate electron beam assisted deposition of carbon protective thin layer (a thin dark region on the top of $\mathrm{AgNW}$ ); white arrow indicates ion beam assisted deposition of carbon protective thick layer; red rectangle indicates area of interest, detailed later in the manuscript.

\section{Results and Discussion}

In Figure 2a, AgNWs are depicted, which have been drop cast on Si substrate and annealed nominally at $250{ }^{\circ} \mathrm{C}$. Welding process between two AgNW takes place due to thermal activation at relatively low temperature (well below the melting point, $\mathrm{T}_{\mathrm{m}}$ ) in three stages, as shown schematically in Figure 3 . This process essentially represents a typical sintering. The morphology of $\mathrm{AgNW}$ welded junctions was investigated in more detail using SEM with images shown in Figures $2 \mathrm{~b}$-d. Histogram of AgNW size distribution, presented as frequency as a function of diameter, is presented in $2 \mathrm{e}$, where the average diameter was calculated to be $132.3 \pm 32.2 \mathrm{~nm}$ with the majority of the nanowires being in the reasonably narrow $100-150 \mathrm{~nm}$ range. 


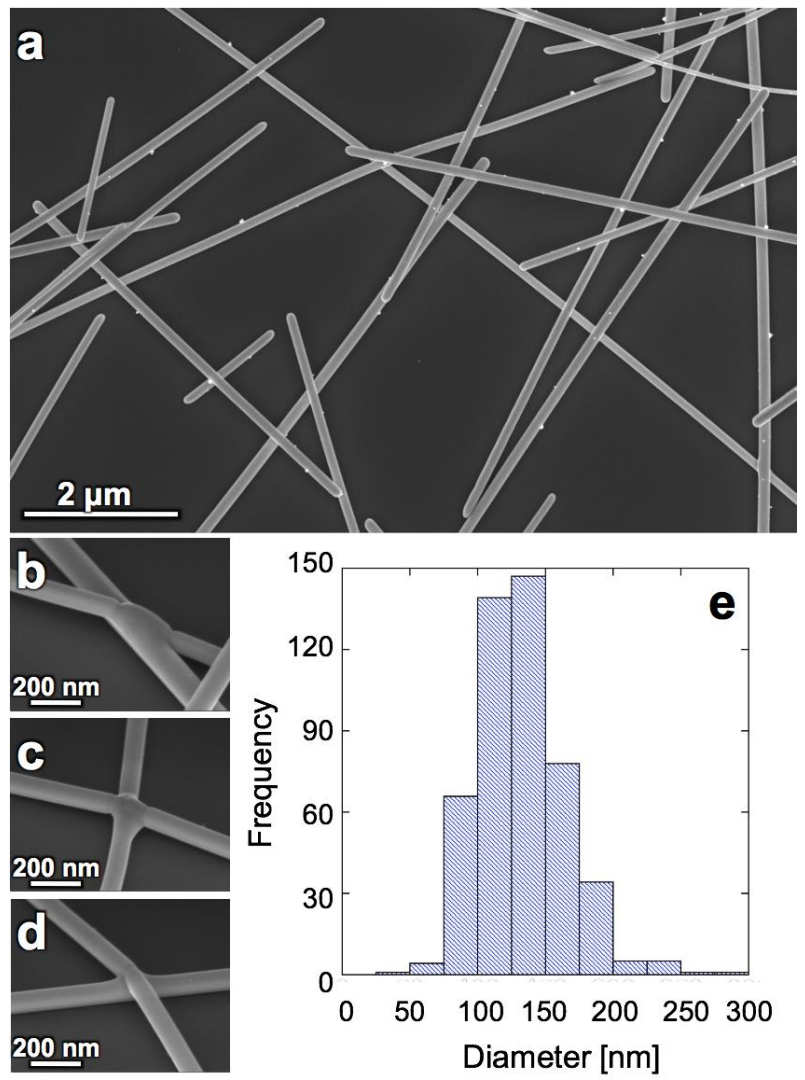

Fig. 2. Scanning electron micrographs of a) annealed AgNW network, $b$-d) random welded junctions at higher magnification, e) histogram of AgNW diameter distribution [22].

When a sharp corner of one nanowire touches a flat 100 type facet of the other nanowire (Figure 3a), due to the Gibbs-Thompson capillary effect [21], dissolution of the sharp corner starts taking place, which leads to surface diffusion of Ag atoms, solid-state wetting and neck formation (Figure $3 b$ ), and finally to the formation of NW welded junctions, as shown in Figure 3c. 


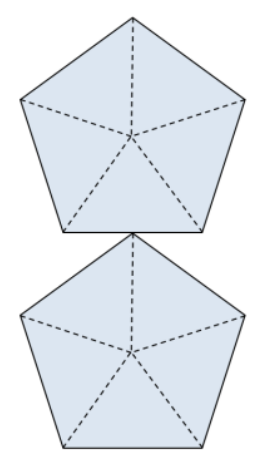

a

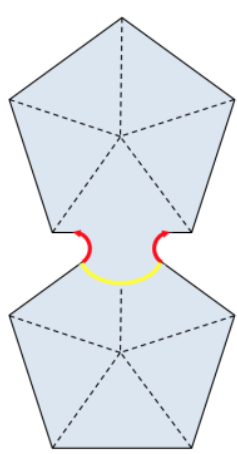

b

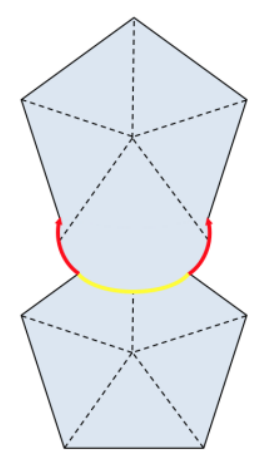

C

Fig. 3. Schematic presentation of the three-stage welding process: a) corner of one nanowire touching flat 100 type facet of another nanowire; b) solid-state wetting and neck formation between nanowires in contact; c) growth of welded zone; (red arrows indicate surface diffusion paths of Ag atoms; yellow lines represent grain boundary created between nanoweld and the NW initially in contact via corner) [22].

This process can be correlated with the Ostwald ripening phenomenon [21], in which the particle with a smaller diameter, feeds additional material to the particle with a larger diameter. In this case, the corners of NWs can be considered to have infinitely small radii of curvature and the facets can be considered to have infinitely large radii of curvature. The driving force for this process is the decrease of the high surface energy of AgNWs in contact. In a solid-solid transformation, the strain energy effect occurs so strain energy change must be included, together with the interfacial energy changes. Free energy $(\Delta G)$ of the system can be expressed through volume free energy, surface and strain terms, as shown in Equation 1.:

$$
\Delta G=-V \cdot \Delta G_{V}+\sum_{i=1}^{n} A_{i} \cdot \gamma_{i}+V \cdot \Delta G_{e}
$$

where $V$ is the molar volume, $\Delta G_{V}$ is the driving force for phase transition per unit volume, $A_{i}$ is the surface/interface area, $\gamma_{i}$ is the surface/interface energy and $\Delta G_{e}$ is the strain energy per unit volume. In order for welding to occur, the total free energy of welded NWs has to be smaller than the free energy of NWs before welding (Equation 2.):

$$
\Delta G_{\text {welded } N W s}<\Delta G_{N W \text { corner }}+\Delta G_{N W \text { facet }}
$$

Where $\Delta \mathrm{G}_{\mathrm{NW} \text { corner }}$ is the free energy of $\mathrm{NW}$ in contact through the corner, while $\Delta \mathrm{G}_{\mathrm{NWfacet}}$ is the free energy of NW in contact through facet. Since the volume free energy term $\left(-V \cdot \Delta G_{V}\right)$ is the same for dissolving $\mathrm{NW}$ and the nanoweld, and no phase transitions are taking place, the free energy of the welded nanowires will depend on surface/interface and volume strain energy terms (such as free surfaces, twin boundaries, stacking faults, dislocations) as shown in Equation 3: 


$$
\begin{gathered}
\sum_{i=1}^{n} A_{i_{\text {welded } N W s}} \cdot \gamma_{i_{\text {welded } N W s}}+V_{\text {welded NWs }} \cdot \Delta G_{e_{\text {welded } N W s}}<\left[\sum_{i=1}^{n} A_{i_{N W}}\right. \\
\left.\gamma_{i_{N W}}+V_{N W} \cdot \Delta G_{e_{N W}}\right]_{\text {corner }}+\left[\sum_{i=1}^{n} A_{i_{N W}} \cdot \gamma_{i_{N W}}+V_{N W} \cdot \Delta G_{e_{N W}}\right]_{\text {facet }}
\end{gathered}
$$

After annealing of AgNWs, a $100 \mathrm{~nm}$ encapsulating layer of aluminum-doped zinc oxide (AZO) was deposited by atomic layer deposition (ALD) [15]. Figure 4a shows scanning electron micrographs of AgNWs encapsulated with $\mathrm{AZO}$, as well as welded junctions in more detail (Figures 4b-d). Histogram of AgNW/AZO size distribution, presented as frequency as a function of diameter, is shown in Figure 4e, where the average diameter was calculated to be $310.4 \pm 38.2 \mathrm{~nm}$ with the majority of the nanowires being in the reasonably narrow $300-325 \mathrm{~nm}$ range.
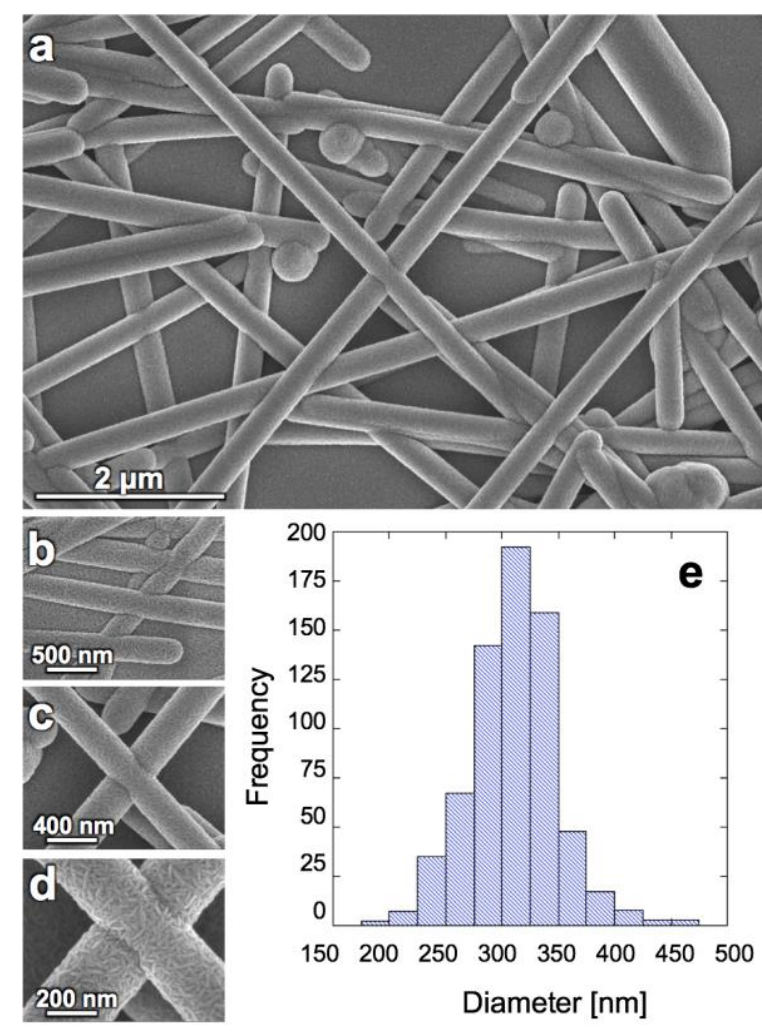

Fig. 4. a) Scanning electron micrographs of a) network of annealed AgNWs after encapsulation by AZO, $b$-d) welded junctions at higher magnification, e) histogram of AgNW/AZO diameter distribution [22].

Figure 5a represents a high resolution phase-contrast image of the AZO layer. Three grains of AZO (noted by numbers 1,2 and 3) with their respective Fast Fourier Transformations (FFTs) shown as insets. Figure $5 \mathrm{~b}$ represents an enlarged FFT of grain 1 while Figure $5 \mathrm{c}$ is a simulated aluminum-doped $\mathrm{ZnO}$ wurtzite structure in [100] zone axis with 010, 002 and 012 reflections. High resolution phase-contrast image (Figure 4a) 
gives evidence of sympathetic nucleation [23] i.e. nucleation of grains of a phase on already formed grains of the same phase. Here, as shown by the crystallographic relationships in the high resolution image, grains exhibit crystallographic co-dependence. An FFT of grain 3 (inset in Figure 5a), gives evidence of 010 spot splitting (noted by yellow arrows), a consequence of the presence of inversion domain boundaries (IDBs) in the $\mathrm{ZnO}$ wurtzite structure $[24,25]$ which can, in some cases, be associated with indium $[26,27]$ but in this case can be the result of doping with aluminum. A single translation interface in $\mathrm{ZnO}$, typical for IDB, with the translation vector of $1 / 3$ [100], will cause spot splitting in the perpendicular direction (noted by arrows in FFT of grain 3). Formation of IDBs is also facilitated by a low stacking fault energy of $43 \mathrm{~mJ} / \mathrm{m}^{2}$ [28] on the basal $\mathrm{ZnO}$ wurtzite plane.

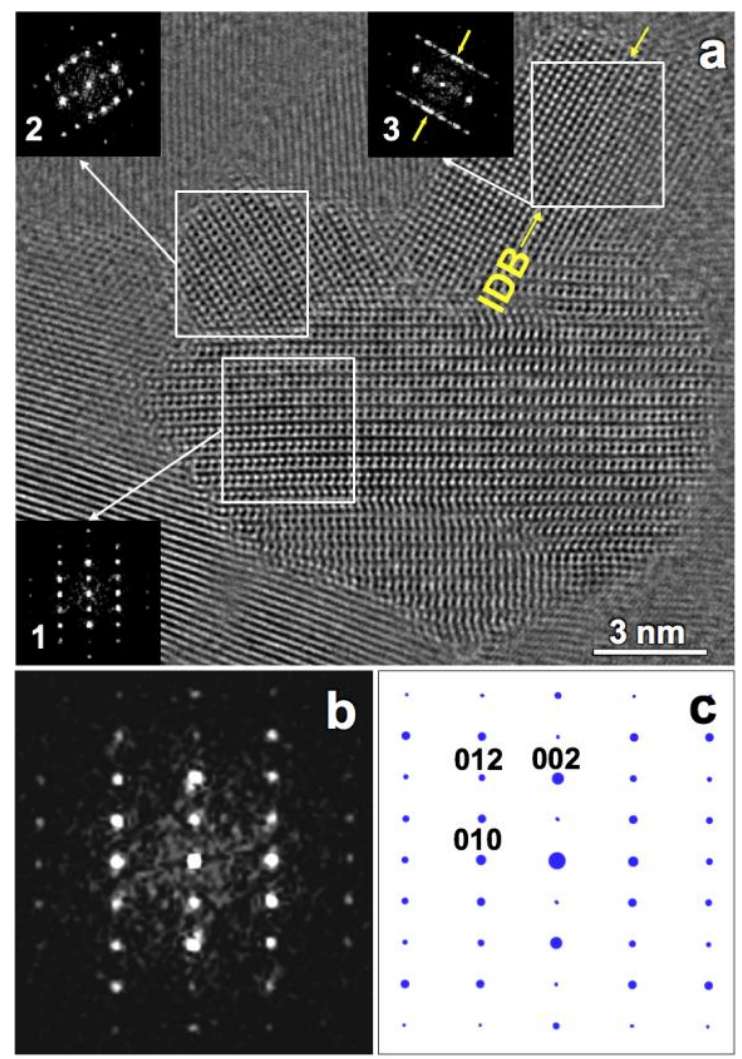

Fig. 5. a) HRTEM of AZO layer taken close to [100] zone axis; insets represent FFTs of grains 1, 2 and 3 taken from areas noted by white squares while yellow arrows note inversion domain boundary (IDB), b) enlarged FFT taken from grain 1 in a), c) Simulated $\mathrm{ZnO}$ wurtzite structure in [100] zone axis with noted reflections [22].

In order to fully elucidate the AgNW microstructure as well as the morphology of the welded zone, a cross-section of the sample was made, as explained in the Experimental section. In the low magnification STEM annular dark field (ADF) crosssectional image (Figure 6a) we can observe AgNW pentagonal structure (white cores) 
and uniform thickness of the AZO layer (grey shells). The AZO uniform growth can also be seen from the Si substrate. With a detailed analysis of a single $\mathrm{AgNW}$, by zooming in the red square region (Figure $5 \mathrm{~b}$ ), we can see non-homogenous intensity distribution inside the $\mathrm{AgNW}$, a result of diffraction and strain contributions. Five white lines, indicated by yellow arrows, represent twin planes dividing the 5 twin segments while white regions inside individual twin segments or along with twin interfaces, represent areas of strain inherent from the synthesis and annealing processes. Basic crystallography explains the origin of this strain. We clearly see that 5 segments, each with a $70.5^{\circ}$ angle between $\{111\}$ twinning planes, fall short $7.5^{\circ}$ of forming a full $360^{\circ}$ circle, or creating compact crystal without strain. So, in order to create a solid structure, the existing atomic planes have to be significantly strained to compensate for the $7.5^{\circ}$ missing angle. The larger this pentagonal crystal grows, the larger the strain becomes [22].

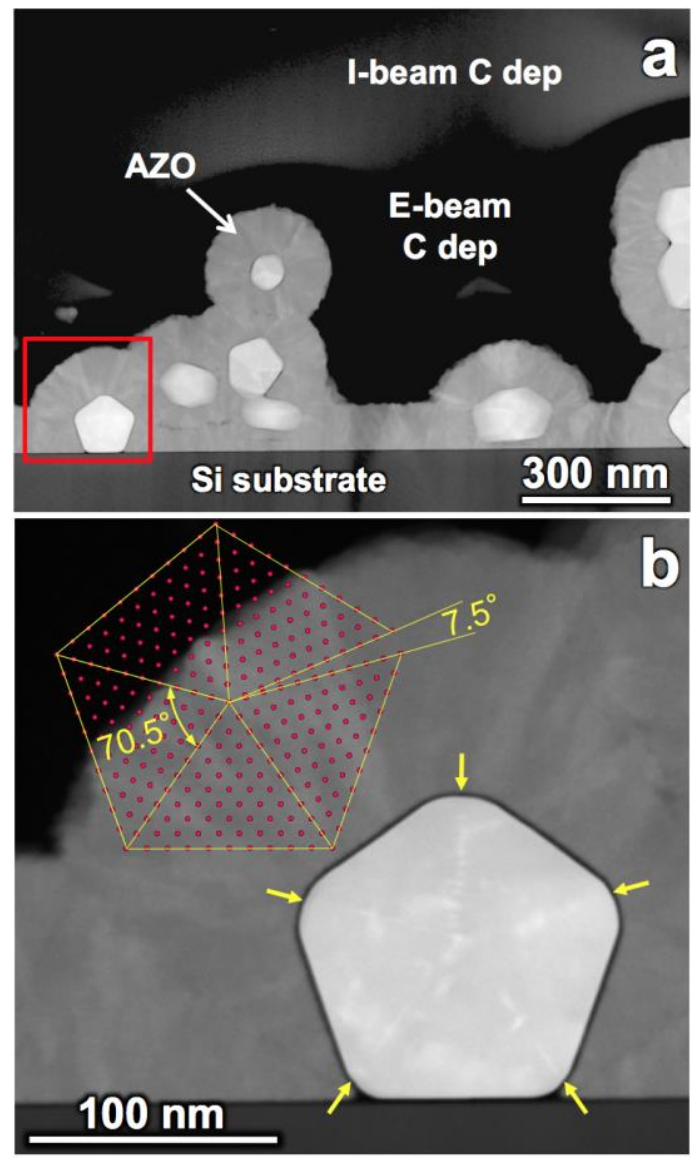

Fig. 6. a) Low magnification ADF STEM of AgNW/AZO nanocomposite; b) Magnified region noted by red square in a); inset is an illustration depicting AgNW cross-section with 5 twin segments, each with a $70.5^{\circ}$ angle between $\{111\}$ twinning planes, which falls short $7.5^{\circ}$ of forming a full $360^{\circ}$ circle; yellow arrows indicate twin planes within AgNW [22]. 
Figure 7, which consists of the STEM ADF image of AgNW cross-section encapsulated AZO, and appropriate energy-dispersive X-ray spectroscopy (EDS) elemental maps, confirms the presence of $\mathrm{Zn}, \mathrm{O}$ and $\mathrm{Al}$ in the shell and $\mathrm{Ag}$ in the core, and no elemental mixing is observed. A certain amount of $\mathrm{Al}$ segregation at the core/shell interface is observed. This could be either due to the processing parameters of the ALD technique, where the initial deposition cycle was of $\mathrm{Al}_{2} \mathrm{O}_{3}$ [15], or more likely, since $\mathrm{Ag}$ is a catalyst for $\mathrm{Al}_{2} \mathrm{O}_{3}$ growth as reported by John et al. [29].
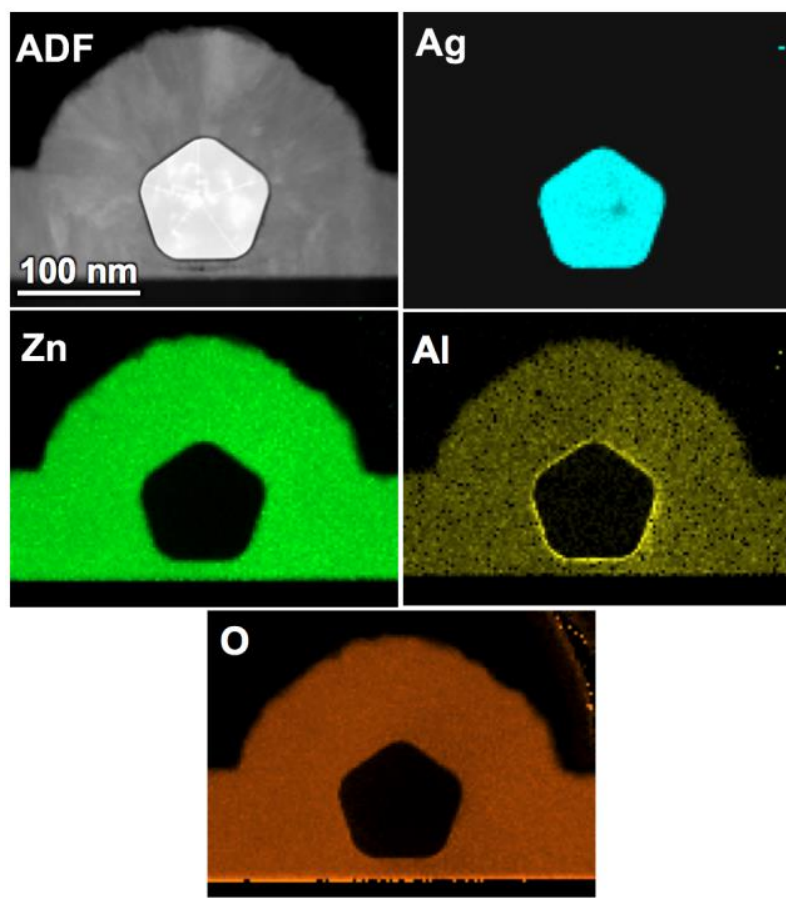

Fig. 7. ADF STEM image and corresponding EDS maps of the elemental distribution of $\mathrm{Ag}, \mathrm{Zn}, \mathrm{Al}$ and $\mathrm{O}$ in the core and the shell [15].

Figure 8 is a high resolution ADF STEM image depicting the area between the two AgNWs in contact through a welded zone. The wetting angle between NW in contact via facet and the welded zone was measured to be $\sim 4.8^{\circ}$, indicating almost complete wetting. The red dotted line represents the twin plane, while solid red lines represent the orientation of $\{111\}$ planes symmetrical to each other with respect to $\{111\}$ twin plane. It is clear that there is no visible distortion of $\{111\}$ planes, parallel to the twin plane, going from the "top" NW into the welded zone. During solid-state wetting and subsequent welding of two AgNWs, the welded zone expands with the incorporation of $\mathrm{Ag}$ atoms into close-packed $\{111\}$ planes. This means that $\mathrm{Ag}$ atoms diffuse along the surface of the NW and are epitaxially attached on the 100 facet of the NW in contact ("top NW"), resulting in $\{111\}$ planes growing in the direction toward the AgNW in contact via corner ("bottom NW"). In these welded AgNWs atoms from one AgNW are epitaxially attached to the other AgNW, and this phenomenon was proposed to be called homoepitaxial growth [22]. With this in mind, the terms NW site specific coarsening or NW sintering 
are also more appropriate for this process than NW welding (as no filler material is used for joining of NWs). However, the term NW welding is used, as consensus has been made in literature [22].

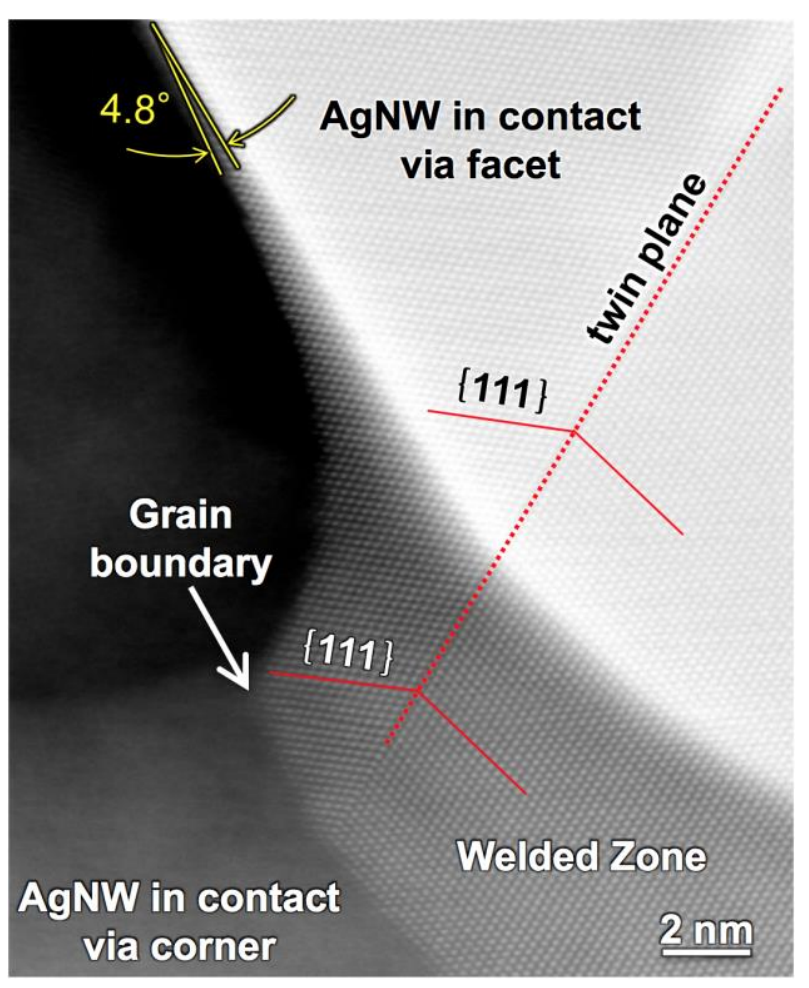

Fig. 8. a) High resolution ADF STEM image of the welded zone between two AgNWs; in the image are noted wetting angle, continuous twin plane and grain boundary between AgNW and welded zone [22].

In Figure 9, a high resolution ADF STEM image of the other side of the same welded zone between two AgNWs is shown. The left inset is a lower magnification image of two AgNWs with a black square denoting the analyzed zone. Inset in Figure $9 \mathrm{a}$ is an FFT from the area denoted by the red square, displaying $\langle 110\rangle$ zone axis. Figure $9 \mathrm{~b}$ presents a magnification of the area indicated by the red square in Figure 9a. This section of nanoweld is characterized by the presence of several kinds of faults: nanotwins (NT), stacking faults (SF) and dislocations (D), as shown in Figure 8c, which represents a moiré pattern obtained using 111 and 200 reflections, indicated by a yellow circle in FFT inset in Figure 9a. Change of orientation of $\{111\}$ planes in the area denoted by NT in moiré pattern is an indication of the presence of 5-layer thick nanotwin in the welded zone and these fringes are obtained using 200 reflection in the yellow circle in FFT shown as the inset in Figure 9a. This nanotwin is bounded by Shockley partial dislocations [30, 31, 32], creating the appearance of an HCP (hexagonal close-packed) local atom arrangement at the FCC AgNW nanotwin interface. 


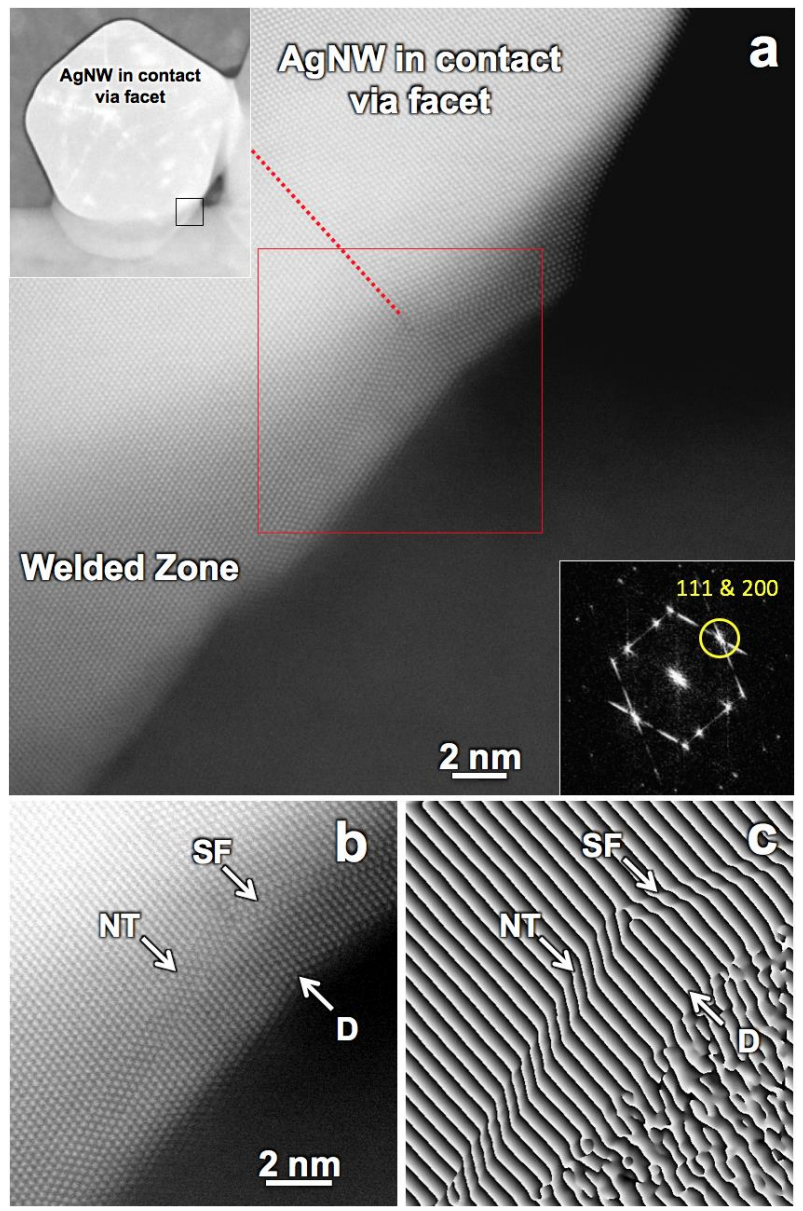

Fig. 9. a) Low magnification high resolution ADF STEM image of area noted by black square in left inset; right inset: FFT from the red square region, b) enlarged region indicated by red square in a); c) moiré pattern obtained using 111 and 200 reflections indicated by yellow circle in FFT inset in a); NT, SF and D denote nanotwin, stacking faults and dislocation, respectively [22].

The formation of nanotwins and stacking faults, seen in Figure 9, can be explained by small stacking fault energy of $\mathrm{Ag}$ of $16 \mathrm{~mJ} / \mathrm{m}^{2}$ [33]. However, an additional possible contribution from strain relaxation shouldn't be ruled out. Preliminary electron tomography data indicate a significant reduction in the volume of NW in contact through the corner, in the vicinity of nanoweld, creating mass balance with the volume of the nanoweld [34]. In addition to nanotwins (NT), the presence of dislocations (D) and stacking faults (SF) (Figures 9b and c) corroborate the fact that this zone is not without defects so a recrystallization process could not have formed it. Analyzing various parts of the welded zone, it was deduced that it is homoepitaxially attached, with crystallographic orientation inherent from AgNWin contact through facet, including twin 
plane extending through nanoweld, associated with the addition of an extra 111 plane in the welded zone, indicating the presence of an edge dislocation (D) [22].

\section{Conclusions}

Welding of two silver nanowires proceeds in three steps: the dissolution of AgNWs with a smaller radius of curvature, solid-state wetting and formation of the neck between AgNWsby surface diffusion, nanoweld growth, where Ag atoms supply is originating from dissolving $\mathrm{AgNW}$. Encapsulation of $\mathrm{AgNW}$ s cores by Al-doped $\mathrm{ZnO}$ shells taking place without elemental mixing. Some segregation of aluminum at the core/shell interface is observed. In order to accommodate significant elastic strain accumulated from the synthesis and annealing/welding, silver nanowires in contact generate defects such as stacking faults, twins and dislocations. The welded zone displays the same crystallographic orientation as the AgNW in contact through the facets to which it is attached.

\section{Acknowledgments}

Vuk V. Radmilović acknowledges support by the Ministry of Education, Science and Technological Development of the Republic of Serbia, under contract No. III45019. Velimir R. Radmilović acknowledges support by the Serbian Academy of Sciences and Arts under contract \#F-141. The authors thank professor Erdmann Spiecker from the Institute of Micro- and Nanostructure Research at the University of Erlangen-Nuremberg, Germany, for the use of experimental facilities.

In memory of Distinguished Professor of Physical and Mechanical Metallurgy, Djordje Drobnjak.

\section{References}

[1] K. Ellmer: Nat Photonics, 6 (2012) 809-817.

[2] A. Bid, A. Bora, A.K. Raychaudhuri: Phys Rev B, 74 (2006) 035426.

[3] L. B. Hu, H. S. Kim, J.Y. Lee, P. Peumans and Y. Cui: ACS Nano, 4 (2010) 2955- 2963.

[4] D. Hecht, L. Hu, G. Gruner: Appl Phys Lett, 89 (2006) 133112.

[5] D. Langley, PhD thesis, Silver nanowire networks: effects of percolation and thermal annealing on physical properties, University of Grenoble, Grenoble, France, 2015.

[6] J-Y. Lee, S.T. Connor, Y. Cui, P. Peumans: Nano Lett, 8 (2008) 689-692.

[7] E.C. Garnett, W. Cai, J.J. Cha, F. Mahmood, S.T. Connor, M.G. Christoforo, Y. Cui, M.D. McGehee, M.L. Brongersma: Nat Mater, 11 (2012) 241-249.

[8] Y. Yang, S. Ding, T. Araki, J. Jiu, T. Sugahara, J. Wang, J. Vanfleteren, T. Sekitani, K. Suganuma: Nano Res, 9 (2016) 401-414.

[9] J.H.M. Maurer, L. Gonzalez Garcia, B. Reiser, I. Kanelidis, T. Kraus: ACS Appl Mater Interfaces, 7 (2015) 7838-7842.

[10] S. Zhu, Y. Gao, B. Hu, J. Li, J. Su, Z. Fan, J. Zhou, Nanotechnology, 24 (2013)

[11] Y. Lu, J.Y. Huang, C. Wang, S. Sun, J. Lou, Nature Nanotechnology, 5 (2010) 218-224

[12] H. Lu, D. Zhang, J. Cheng, J. Liu, J. Mao, W.C.H. Choy: Adv Funct Mater, 25 (2015) 4211-4218. 
[13] U. Dahmen, C.J.D. Hetherington, V.R. Radmilovic, E. Jonson, S.Q. Xiao, C.P. Luo, K.H. Westmacott: Microsc Microanal, 8 (2002) 247-256.

[14] V.V. Radmilovic, J. Kacher, E.R. Ivanovic, A.M. Minor, V.R. Radmilovic: Cryst Growth Des, 16 (2016) 467 - 474

[15] M. Goebelt, R. Keding, S.W.Schmitt, B. Hoffmann, S. Jaeckle, M. Latzel, V.V. Radmilovic, V.R. Radmilovic, E. Spiecker, S. Christiansen: Nano Energy, 16 (2015) 196-206.

[16] V.V. Radmilovic, M. Goebelt, S. Christiansen, E. Spiecker, V.R. Radmilovic: Nanotechnology, 28 (2017) 385701.

[17] K.E. Korte, S.E. Skrabalak, Y. Xia: J Mater Chem, 18 (2008) 437.

[18] C. Ducamp-Sanguesa, R. Herrera-Urbina, M. J. Figlarz: J Solid State Chem, 100 (1992) 272-280.

[19] Y. Sun, B. Mayers, T. Herricks, and Y. Xia: Nano Lett, 3(2003) 955-960.

[20] S. Coskun, B. Aksoy, H.E. Unalan: Cryst Growth Des, 11 (2011) 4963-4969.

[21] D.A. Porter, K.E. Easterling, "Phase transformations in metals and alloys", 2nd ed., Chapman and Hall, London, UK 1992, p. 315.

[22] V.V. Radmilovic, PhD Thesis, Transparent nanocomposite films for plastic electronics applications, University of Belgrade, Faculty of Technology and Metallurgy, Serbia, 2016.

[23] H.I. Aaronson, G. Spanos, R.A. Masamura, R.G. Vardiman, D.W. Moon, E.S.K. Menon, M.G. Hall: Mater Sci Eng B, 32 (1995) 107-123.

[24] D. Van Dyck, G. Van Tendeloo, S. Amelinckx: Ultramicroscopy, 15 (1984) 357 370.

[25] S. Li, H. Lei, Z. Wang, J. Chen, P. Ruterana: Phys Status Solidi B, 255 (2018) 1700429.

[26] Y. Yan, S.J. Pennycook, J. Dai, R.P.H. Chang, A. Wang, T.J. Marks: Appl Phys Lett, 73 (1998) 2585-2587.

[27] M.A. McCoy, R.W. Grimes, W.E. Lee: Philos Mag A, 76 (1997) 1187-1201.

[28] S. Takeuchi, K. Suzuki, K. Maeda, H. Iwanaga: Philos Mag A, 50 (1985) 171178.

[29] J.F. John, S. Mahurin, S. Dai, M.J. Sepaniak: J Raman Spectrosc, 41 (2010) 411.

[30] K.A. Afanasyev, F. Sansoz: Nano Lett, 7 (2007) 2056-2062.

[31] T. Filleter, S. Ryu, K. Kang, J. Yin, R.A. Bernal, K. Sohn, S. Li, J. Huang, W. Cai, H.D. Espinosa: Small, 8 (2012) 2986-2993.

[32] L. Liu, D. Shen, G. Zou, P. Peng, Y. Zhou: Scr Mater, 114 (2016) 112-116.

[33] R.E. Smallman, R.J. Bishop, Modern Physical Metallurgy and Materials Engineering, Butterworth-Heinemann, Oxford, UK 1999, p. 101.

[34] V.V. Radmilovic, P. Ercius, V.R. Radmilovic, 2020., manuscript in preparation.

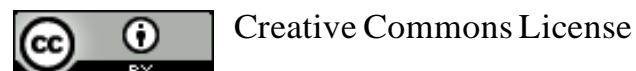

This work is licensed under a Creative Commons Attribution 4.0 International License. 